\title{
A novel GATA6 mutation in patients with tetralogy of Fallot or atrial septal defect
}

\begin{abstract}
Xiaoping Lin ${ }^{1,2,3,7}$, Zhaoxia Huo ${ }^{2,3,4,7}$, Xingyuan Liu ${ }^{5,7}$, Yangyang Zhang ${ }^{6,7}, \mathrm{Li} \mathrm{Li}^{2,3}$, Hong Zhao ${ }^{5}$, Biao Yan $^{4}$, Ying Liu ${ }^{4}$, Yiqing Yang ${ }^{1,2,3}$ and Yi-Han Chen ${ }^{1,2,3}$

GATA6 is a member of the GATA family of transcription factors, and its expression and functions overlap with those of GATA4 during heart development. Mutations in GATA4 have been related to human congenital heart diseases (CHDs) in several studies, whereas mutations in GATA6 have only recently been reported in patients with persistent truncus arteriosus. Animal experiments have revealed critical roles for GATA6 in the development of the myocardium and cardiac morphogenesis, thereby highlighting the potential involvement of GATA6 defects in the pathogenesis of CHDs. Here, we screened the GATA6 in 270 individuals with sporadic CHDs by direct sequencing. After identification of the mutation, a luciferase reporter assay and real-time quantitative polymerase chain reaction were performed to detect functional changes in the mutant transcription factor. The same heterozygous missense mutation (Ser184Asn) was identified in three patients, including one with tetralogy of Fallot and two with atrial septal defects. This mutation was not found in $\mathbf{5 0 0}$ unrelated ethnically matched healthy subjects. Direct sequencing of this region in the parents of these three patients revealed the same mutation in one of the parents for each patient, and one of the parent carriers presented with a bicuspid aortic valve. Biological analysis revealed clearly decreased transcriptional activity of GATA6 Ser184Asn in vitro. All these data suggest that GATA6 Ser184Asn is a novel mutation associated with CHDs and has an important role in disease pathogenesis.
\end{abstract}

Journal of Human Genetics (2010) 55, 662-667; doi:10.1038/jhg.2010.84; published online 15 July 2010

Keywords: congenital heart disease; GATA6; genetics; mutation; transcriptional factor

\section{INTRODUCTION}

Congenital heart disease (CHD) is present in 19-75 of every 1000 live births and constitutes a major percentage of clinical birth defects. ${ }^{1}$ It is also considered to be a leading cause of morbidity and mortality in infants. CHD arises from abnormal heart development during embryogenesis and is thought to be partly caused by genetic defects. ${ }^{2}$ Recent studies have identified a series of genes that contribute to inherited and sporadic CHD, and most of these genes encode cardiac transcription factors such as Nkx2.5, TBX5 and GATA4. ${ }^{3-6}$

GATA family members (GATA1-6) contain two zinc-finger domains that bind to a consensus site, (A/T) GATA (A/G), and mediate interactions with diverse factors. ${ }^{7}$ GATA4, 5 and 6 are expressed early in the heart tissue and endodermal derivatives. ${ }^{7,8}$ Of these three GATA transcription factors, GATA4 has been most extensively studied and is thought to have a dominant role in heart development. ${ }^{9}$ Numerous mutations in GATA4 have been recognized in a wide range of cases, including tetralogy of Fallot (TOF), pulmonary stenosis, atrial septal defects (ASDs), ventricular septal defects, atrioventricular septal defects and patent ductus arteriosus. ${ }^{4,10-17}$ GATA6 is another member of the GATA family with expression and functions that overlap with GATA4 during cardiovascular development. ${ }^{7}$ Recent experiments in animals have revealed critical roles for GATA6 in the development of the myocardium and cardiac morphogenesis, highlighting the potential involvement of GATA6 mutations in the pathogenesis of human CHD. ${ }^{18-21}$ Although mutations in GATA6 have recently been related to patients with persistent truncus arteriosus, their involvement in, and impact on, various types of CHD are unclear. ${ }^{22}$

To explore this question, we investigated 270 individuals with sporadic CHD for GATA6 mutations and identified the biological functional deficits of an identified mutation in vitro.

\section{MATERIALS AND METHODS}

Study population

A total of 270 unrelated patients with sporadic CHD (Table 1) were identified in the Chinese population. Peripheral venous blood specimens were obtained,

${ }^{1}$ Department of Cardiology, East Hospital, Tongii University School of Medicine, Shanghai, PR China; ${ }^{2}$ Key Laboratory of Arrhythmias, Ministry of Education, China (Tongji University), Shanghai, PR China; ${ }^{3}$ Institute of Medical Genetics, Tongji University, Shanghai, PR China; ${ }^{4}$ Department of Biochemistry and Molecular Biology, School of Life Science and Technology, Tongji University, Shanghai, PR China; ${ }^{5}$ Department of Pediatrics, Tongji Hospital, Tongii University School of Medicine, Shanghai, PR China and ${ }^{6}$ Department of Cardiology, First Affiliated Hospital of Nanjing Medical University, Nanjing, PR China

${ }^{7}$ These authors contributed equally to this work.

Correspondence: Professor Y-H Chen, Key Laboratory of Arrhythmias, Ministry of Education, China (Tongji University), 389 Xin Cun Road, Shanghai 200065, PR China. E-mail: yihanchen@tongji.edu.cn

Received 25 March 2010; revised 10 June 2010; accepted 14 June 2010; published online 15 July 2010 
Table 1 Characteristics of study population with CHD

\begin{tabular}{|c|c|}
\hline Phenotype & $\begin{array}{c}\text { Number of } \\
\text { patients }\end{array}$ \\
\hline Isolated CHD & 235 \\
\hline $\mathrm{VSD}^{\mathrm{a}}$ & 33 \\
\hline $\mathrm{ASD}^{\mathrm{b}}$ & 163 \\
\hline TOF & 20 \\
\hline TAPVC & 1 \\
\hline TGA & 1 \\
\hline PDA & 5 \\
\hline PS & 2 \\
\hline DORV & 3 \\
\hline $\mathrm{COA}$ & 1 \\
\hline CAVC & 1 \\
\hline PAPVC & 3 \\
\hline Cor Triatriatum & 1 \\
\hline RVOTO & 1 \\
\hline Complex $C H D$ & 35 \\
\hline VSD/PDA & 5 \\
\hline ASD/NSD & 14 \\
\hline PFO/VSD & 4 \\
\hline PFO/VSD/PDA & 2 \\
\hline DORV/NSD & 3 \\
\hline DORV/ASD/VSD & 1 \\
\hline COA/PDA & 1 \\
\hline TGA/VSD & 3 \\
\hline ASD/VSD/PDA & 1 \\
\hline ASD/PDA & 1 \\
\hline Total & 270 \\
\hline \multicolumn{2}{|c|}{$\begin{array}{l}\text { Abbreviations: ASD, atrial septal defect; CAVC, common arteriovenous canal; CHD, congenital } \\
\text { heart disease; COA, coarctation of the aorta; DORV, double outlet right ventricle; PAPVC, partial } \\
\text { anomalous pulmonary venous connection; PDA, patent ductus arteriosus; PFO, patent foramen } \\
\text { ovale; PS, pulmonary stenosis; RVOTO, right ventricular outflow tract obstruction; TAPVC, total } \\
\text { abnormal pulmonary venous connection; TGA, transposition of great arteries; TOF, tetralogy of } \\
\text { Fallot; VSD, ventricular septal defect. } \\
\text { aPerimembranous, membranous, muscular ventricular septal defect. } \\
\text { bOstium secundum, sinus venosus atrial septal defect. }\end{array}$} \\
\hline
\end{tabular}

and clinical data including medical records, electrocardiogram, echocardiography and cardiac catheterization reports were systematically reviewed. Most of the recruited patients (102 females and 168 males) in this study underwent cardiac operations or catheterization. Patients with syndromic CHD such as Marfan, Noonan, Holt-Oram, Alagille and CHARGE syndromes as well as chromosomal abnormalities that are highly associated with CHD, such as 21 trisomy and 22q11.2 deletion, were excluded from this study. The controls were initially 300 unrelated ethnically matched healthy subjects who did not have CHD and were later extended to 500. All study subjects were of Chinese Han nationality. Genomic DNA from all participants was extracted from blood lymphocytes using a Wizard Genomic DNA Purification Kit (Promega, Madison, WI, USA). The study was performed according to the terms required by the Research Ethics Committee of Tongji University School of Medicine, and written informed consent was obtained from all participants.

\section{Genetic investigations}

Primers to amplify the complete coding region and exon/intron boundaries of the GATA6 gene (NM_005257) were designed with Primer 3 software online (Table 2). Polymerase chain reaction (PCR) was performed using HotStar Taq DNA Polymerase (Qiagen GmbH, Hilden, Germany) with an annealing temperature of $62{ }^{\circ} \mathrm{C}$. The amplified products were purified with a QIAquick gel extraction kit (Qiagen). Both strands of each PCR product were sequenced with a DYEnamic ET dye terminator kit (GE Healthcare, Amersham Place,
Table 2 Primers for GATA6

\begin{tabular}{lll}
\hline Exon & \multicolumn{1}{c}{ Forward $\left(5^{\prime}\right.$ to $\left.3^{\prime}\right)$} & \multicolumn{1}{c}{ Reverse $\left(5^{\prime}\right.$ to $3^{\prime}$ ) } \\
\hline Exon 2-a & TGCAGCCTACGCTCTTGTTA & CTGCAGGTGGTACGGTAGG \\
Exon 2-b & CGGAGGAGATGTACCAGACC & GAAAACAGGGCCCGAGTG \\
Exon 3 & ATAACCCTGCTCCTGGCTTT & ATGGGGAAAGGCTATCTTGG \\
Exon 4 & TTGTTGATGACAGGGACAAAA & TGTTTCCTACTGGTGCATAAGG \\
Exon 5-6 & GGATTAGAGGCGTGAGC & GAACTGTTGGTTTAGATTATTGT \\
Exon 7 & CACAGACCCCGTTCATTAGC & TCCCTTCCATCTTCTCTCA \\
\hline
\end{tabular}

Buckinghamshire, UK) using a MegaBACE 500 DNA Sequencing system (GE Healthcare).

\section{Sequence comparison}

The GATA6 protein sequences from various species were aligned using ClustalW software.

\section{Plasmids and site-directed mutagenesis}

The pcDNA3-hGATA6 plasmid was kindly provided by Edwards-Ghatnekar, Angela V, Division of Rheumatology and Immunology, Medical University of South Carolina. The identified point mutation was introduced into a wild-type GATA6 clone using a QuikChange II XL Site-Directed Mutagenesis Kit (Stratagene, La Jolla, CA, USA). The clones were sequenced to confirm the desired mutation and to exclude any other sequence variations. Flag-tagged plasmids were then prepared for western blot analysis.

\section{Luciferase assays}

HEK-293 cells were transiently transfected with $50,100,200$ or 400 ng of wildtype or mutant pcDNA3-hGATA6; $400 \mathrm{ng}$ of atrial natriuretic factor (ANF) luciferase reporter (-638) (kindly provided by Vidu Garg, Department of Pediatrics, UT Southwestern Medical center); and $40 \mathrm{ng}$ of pGL4.75[hRluc/ CMV] (Promega) using Polyfect Transfection Reagent (Qiagen). The pGL4.75 [hRluc/CMV] plasmid was introduced as an internal control. Firefly luciferase and Renilla luciferase activities were measured with the Dual-Glo luciferase assay system (Promega) at $48 \mathrm{~h}$ after transfection. Three independent experiments were performed at minimum for wild-type and mutant GATA6. The protein expression level was evaluated by western blot analysis.

\section{Real-time quantitative PCR analysis}

H9c2 rat cardiomyoblast cells were transfected with $400 \mathrm{ng}$ of wild-type or mutant pcDNA3-hGATA6 or pcDNA3 control. Total RNA was isolated from H9c2 cells using Trizol reagent (Invitrogen, Carlsbad, CA, USA) at $48 \mathrm{~h}$ after transfection. Reverse-transcription PCR was performed using SuperScript II reverse transcriptase (Invitrogen) with oligo-dT priming according to the manufacturer's instructions. Real-time quantitative PCR was performed with the Mx3000P system (Stratagene) using Power SYBR Green PCR Master Mix (Applied Biosystems, Foster City, CA, USA). GAPDH was chosen as an internal control. Quantitative PCR primers for ANF, brain natriuretic factor, $\beta$-myosin heavy chain and GAPDH were as described earlier. ${ }^{23}$

\section{Statistics}

Data are given as mean \pm s.d. Differences between the two groups were compared using Student's $t$-test for continuous variables and $P$-value $<0.05$ was defined as being statistically significant.

\section{RESULTS}

\section{Identification of a GATA6 mutation in CHD patients}

We identified the same heterozygous missense mutation in the GATA6 gene in three CHD patients, including one with TOF and two with ASDs (Figure 1a). One of the ASD patients was a 3-year-old girl with an ostium secundum ASD $(2.0 \mathrm{~cm})$ and mild pulmonary arterial 


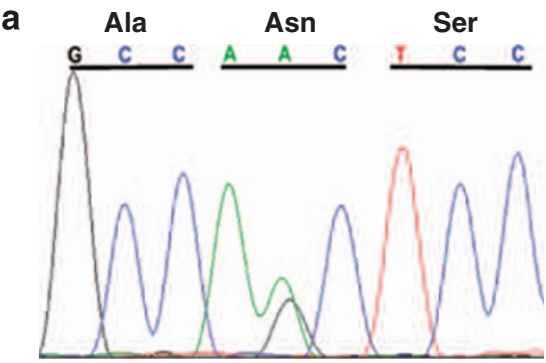

Ser184Asn
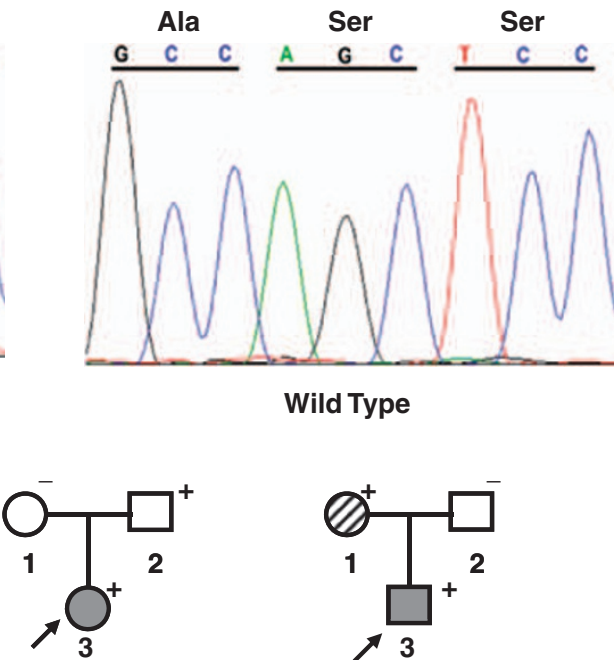

C

b

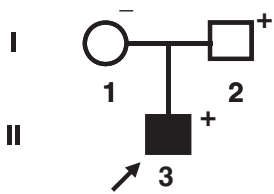

Ser184Asn

$\star$

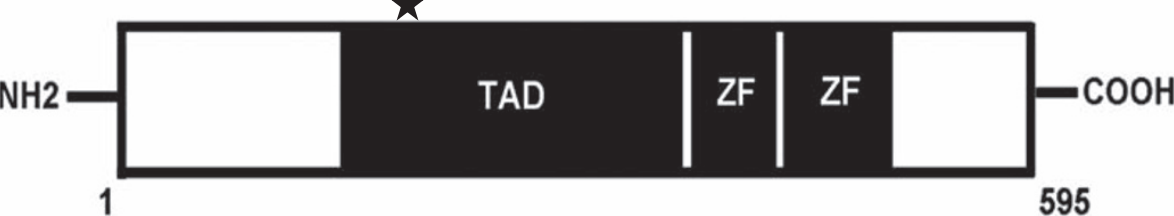

d

151

S184N

Human LAALSSOGPAAYDGAPGG-EVHSAAAAAAAAAAA ISPVYVPTTRVGSML?

Mouse LAALSSQGPAAYDGAPGG-FVHSAAAAAAAAAAA 1 SPVYVPTTRVGSMLS

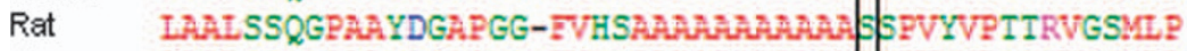

Pig LAALSSQGPAAYDGAPGG-FVHSAARAAARAAAA S SPVYVPTTRVGSMLP

Chicken LAISASQGPAPYDGSPGG-FMHSAP--.--.-- ESPVYVPTRVGSVLP

Frog LIITAAQGPLGYDPSPGT-FMHSAA--.-.- SSPYVPTSRVGSMLI

Zebrafish LAIAAAQSQAGYNDTPSAGYMHSNP-------- IEPVYVPTSRVGTMIP

Figure 1 (a) A mutation was identified in GATA6 in three CHD patients. Ser184Asn and its wild-type counterpart are shown. (b) Pedigree structures of three families with congenital heart disease. Family members are identified by generations and numbers: squares indicate male members; circles indicate female members; solid dark symbols indicate TOF patients; clear symbols indicate unaffected members; gray symbols indicate ASD patients; diagonal symbols indicate BAV patients; arrows indicate probands; ' + ' indicate carriers of the mutation (Ser184Asn); '-' indicate non-carriers; on the left is the TOF boy's family; middle is the ASD girl's family and right is the ASD boy's family. ASD, atrial septal defect; BAV, bicuspid aortic valve; TOF, tetralogy of Fallot. (c) The Ser184Asn mutation was located in the transcriptional activation domain (TAD) of the GATA6 protein. * represents the location of the mutation; ZF, zinc finger. (d) A multiple alignment of GATA6 protein sequences from various species indicated that Ser184Asn was located in a conserved site across mammals.

hypertension. The other ASD patient was a 4-year-old boy with an ostium secundum ASD $(0.33 \mathrm{~cm})$ and a mild tricuspid valve and pulmonary valve replacement. The third patient was a 7-month-old boy with an overriding aorta (50\% override), pulmonary stenosis, a ventricular septal defect and right atrial and ventricular hypertrophy. None of the patients had any other abnormalities. The same substitution was also detected in the father of the TOF boy, the father of the ASD girl and the mother of the ASD boy. The three probands declared no family history of $\mathrm{CHD}$, but echocardiography showed that the mother of the ASD boy had a bicuspid aortic valve (BAV). Her heart was of normal shape and function, and no clinical symptoms were found. The pedigree structures of the three families are illustrated in Figure 1b. The same mutant allele was not detected in the other 267 non-syndromic CHD patients or in 500 ethnically matched healthy controls $(P=0.04$, Fisher's exact test). The identified mutation $(\mathrm{c} .551 \mathrm{G} \rightarrow \mathrm{A})$ predicted the replacement of serine (Ser, S) with asparagine (Asn, N) at codon 184 (Ser184Asn) located in the transcriptional activation domain (TAD) of the GATA6 protein (Figure 1c). A cross-species alignment of GATA6 protein sequences showed that the altered amino acid (Ser184Asn) was located in a region adjacent to a poly(Ala) insertion in mammals that was not present in other vertebrates (Figure 1d). The high conservation of this region across mammals may suggest a unified function in mammals that is different between mammals and other vertebrates.

Our study also revealed one previously reported sequence variant $($ c. $43 \mathrm{G} \rightarrow \mathrm{C})$ predicting an amino acid change at codon 15 (Gly15Arg). ${ }^{22}$ This nucleotide change was found in 7 of 270 nonsyndromic CHD patients (2.6\%) and in 9 of 300 subjects in the control population $(3.0 \%)$. Four other sequence variants in introns and the $3^{\prime}$ UTR of GATA6 were found in both CHD patients and control subjects. Three of these sequence variants have been previously reported in the dbSNP database, and no significant differences were 
Table 3 Sequence variants identified in this study

\begin{tabular}{|c|c|c|c|c|c|}
\hline \multirow[b]{2}{*}{ Location } & \multirow[b]{2}{*}{ Nucleotide change } & \multirow[b]{2}{*}{ dbSNP database } & \multirow[b]{2}{*}{ Amino acid change } & \multicolumn{2}{|c|}{ Allele frequencies } \\
\hline & & & & Patients & Controls \\
\hline Exon 2 & c. $43 \mathrm{G} \rightarrow \mathrm{C}$ & & Gly15Arg & (0.026) 7/270 & (0.033) 10/300 \\
\hline Exon 2 & c. $551 \mathrm{G} \rightarrow \mathrm{A}$ & & Ser184Asn & (0.011) 3/270 & (0.000) 0/500 \\
\hline Intron 2 & c. $1336-85 \mathrm{C} \rightarrow \mathrm{T}$ & & & (0.011) 3/270 & (0.016) 5/300 \\
\hline Intron 2 & c. $1336-60 \mathrm{C} \rightarrow \mathrm{T}$ & rs3764504 & & (0.011) 3/270 & (0.067) 2/300 \\
\hline 3'-UTR & c. $+72 \mathrm{G} \rightarrow \mathrm{A}$ & rs1941084 & & (0.319) 86/270 & 90/300 \\
\hline 3'-UTR & c. $+77 \mathrm{G} \rightarrow \mathrm{A}$ & rs1941083 & & (0.130) 35/270 & (0.193) 58/300 \\
\hline
\end{tabular}

observed between the CHD patients and control subjects in any of their allele frequencies. All sequence variants and their allele frequencies are summarized in Table 3.

\section{Biological study of the identified GATA6 mutation}

The Ser184Asn mutation was located in a conserved region in the TAD of the GATA6 protein. An earlier study of GATA4 indicated that mutations in the TAD region might influence transcriptional activity without affecting DNA-binding affinity or nuclear localization. ${ }^{15}$ Therefore, we examined the transcriptional activation ability of GATA6 in HEK-293 cells using one of its direct cardiac downstream targets, ANF, as a luciferase reporter. Overexpression of wild-type GATA6 activated the ANF promoter in a dose-dependent manner, whereas mutant GATA6 showed no signs of this ability and had significantly deceased transcriptional activity. This finding suggests reduced activity and a loss of dosage sensitivity for GATA6 Ser184Asn in this system (Figure 2a). Western blot analysis indicated no obvious difference in protein levels between wild-type and mutant GATA6 (Figure $2 b$ ).

Additional experiments were performed with a second cell line derived from embryonic heart tissue (H9c2 rat cardiomyoblast cells) using real-time quantitative PCR. Although transcripts of ANF, brain natriuretic factor and $\beta$-myosin heavy chain were upregulated in $\mathrm{H} 9 \mathrm{c} 2$ cells transfected with wild-type GATA6 or GATA6 Ser184Asn compared with the pcDNA3 control (Figure 3), the levels of these target genes were more significantly increased in $\mathrm{H} 9 \mathrm{c} 2$ cells transfected with wild-type GATA6, indicating impaired transcriptional ability of GATA6 Ser184Asn in cardiomyocytes.

\section{DISCUSSION}

We initially identified the same novel GATA6 mutation, Ser184Asn, in three patients with CHD, including two ASD patients and one TOF patient. Further genetic analysis revealed that the Ser184Asn mutation was present in one of the parents for each patient. Echocardiography showed that the mother of the male ASD patient had BAV, but the other two parent carriers displayed no structural cardiac abnormalities, suggesting moderate penetrance of this mutation. The reason for this incomplete penetrance was not clear. The overlapping function of GATA6 with GATA4 (discussed below) or environmental and/or epigenetic factors may lead to different phenotypes. The identified mutation altered a serine residue at position 184 located in the TAD region of the GATA6 protein, which is highly evolutionarily conserved across mammals. Biological activity analysis showed that GATA6 Ser184Asn had clearly decreased transcriptional activity on downstream cardiac genes in both HEK-293 cells and H9c2 cells that mimic cardiomyocytes, indicating that it could contribute to the pathogenesis of CHD.

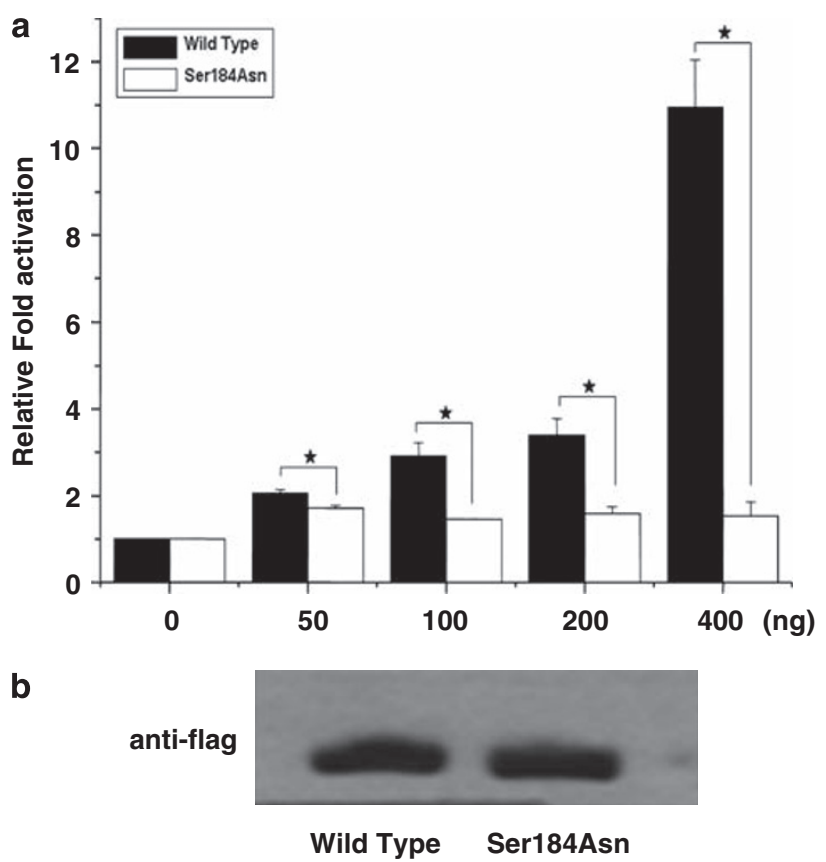

Figure 2 (a) HEK-293 cells were transfected with either 50, 100, 200 or $400 \mathrm{ng}$ of wild-type or mutant GATA6 and $400 \mathrm{ng}$ of an ANF luciferase reporter. The results are shown as the fold activation caused by wild-type or mutant GATA6. In all cases, the data represent the mean \pm s.d. of three independent experiments performed in duplicate. * represents a significant difference in luciferase activity between the wild-type and mutant GATA6 constructs $(P<0.05)$. (b) The protein levels of wild-type and mutant GATA6 were evaluated by western blot analysis after HEK-293 cells were transfected with $400 \mathrm{ng}$ of either flag-tagged plasmid. No obvious differences were observed between the two groups.

Conditional inactivation of GATA6 in vascular smooth muscle cells and cardiac neural crest cells results in a spectrum of malfunctions affecting the cardiac outflow tract and aortic arch in mice. ${ }^{18}$ Furthermore, mutations in the GATA6 zinc-finger and nuclear localization domains give rise to a failure of transactivating target semaphorinplexin pathway genes, leading to cardiac outflow tract defects in humans. $^{22}$ Compared with the above mutations, the mutation found in this study was located in the N-terminal TAD region, which has not been proved to affect DNA-binding affinity or nuclear localization. However, decreased transcriptional activity of GATA6 Ser184Asn on certain downstream cardiac genes was also observed in vitro. It would be interesting to further examine whether this mutation show the same mechanism of alternative regulatory activity 


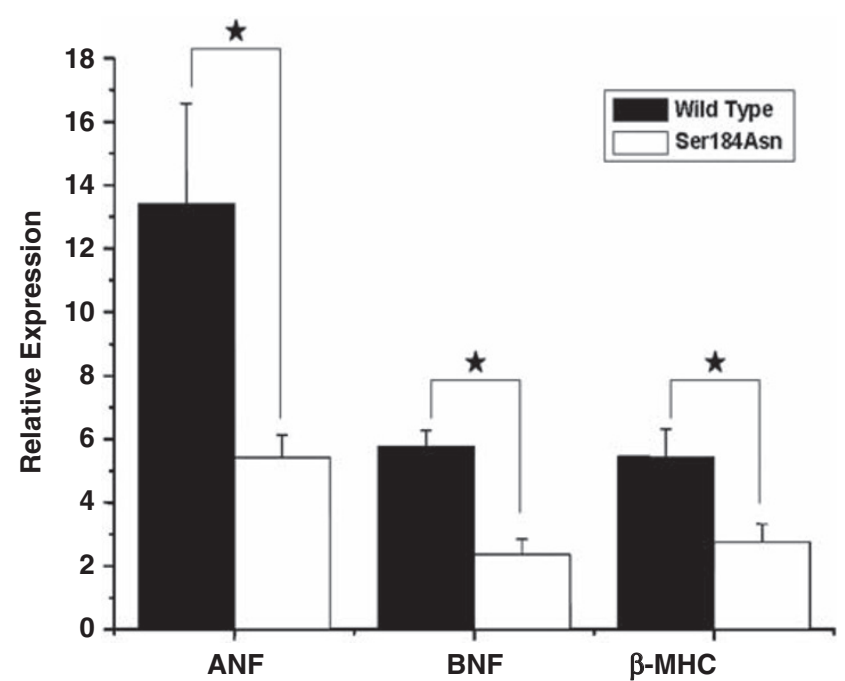

Figure $3 \mathrm{H} 9 \mathrm{c} 2$ cells were transfected with $400 \mathrm{ng}$ of wild-type or mutant GATA6 of the pcDNA3 control. The levels of ANF, brain natriuretic factor (BNF) and $\beta$-myosin heavy chain ( $\beta-M H C)$ mRNA were evaluated by realtime quantitative $P C R$, and the values were normalized to the pcDNA3 control. In all cases, the data represent the mean \pm s.d. of three independent experiments performed in triplicate. * represents a significant difference in mRNA expression levels between the wild-type and mutant GATA6 groups $(P<0.05)$

on the semaphorin-plexin pathway that may lead to the outflow tract defects, in this case, TOF.

$\mathrm{BAV}$ is a common abnormality found in $>1 \%$ of the general population, ${ }^{24}$ suggesting that poor corelations might exist between GATA6 mutation and BAV in ASD boy's mother. However, an earlier study showed that cardiac neural crest cells contribute to the maturation of the aortic valve; thus, dysfunction of the semaphorin-plexin pathway caused by this GATA6 mutation may indirectly undermine the development of the aortic valve by targeting cardiac neural crest cells. ${ }^{25}$ Interestingly, BAV has also exhibited linkage to chromosome $18 \mathrm{q}$, where the GATA6 gene is located. ${ }^{26}$ The above evidences suggested the potential role of GATA6 in the pathogenesis of BAV which, we believe, needs to be further explored in a large cohort of BAV patients.

Compound heterozygosity of GATA4 and GATA6 leads to embryonic lethality because of a spectrum of cardiovascular malfunctions, indicating that these two GATA transcription factors cooperatively and synergistically regulate downstream cardiac genes and that there exists a threshold for both factors to function normally in heart development. ${ }^{20,21}$ Therefore, we also screened GATA4 in the three patients with the Ser184Asn mutation, but no non-synonymous variants were found, excluding the possibility of dual-GATA transcription factor defects in the pathogenesis of the CHD patients in our study. Relatively conserved TAD regions between GATA4 and GATA6 partially explains their certain functional redundancy and the similarities in transcriptional activation mechanisms. ${ }^{27}$ Mutations in GATA4 TAD regions have been related to septal defects in different studies. ${ }^{10,17}$ This study found that the identified mutation in GATA6 TAD region was also associated with ASDs, further supporting the point of a redundant role between GATA4 and GATA6 in cardiogenesis as mentioned earlier. $^{7,20,21,27,28}$ A disrupted interaction between GATA4 and TBX5 is involved in ASDs in both humans and mice. ${ }^{4,19}$ Similarly, a link between GATA6 and TBX5 has been recognized, and compound heterozygosity of both genes results in defects in myocardial development. ${ }^{19}$ Although no direct interactions have been reported between GATA6 TAD region and other transcriptional factors, such as GATA4 and TBX5, the general interactions between those transcriptional factors may aid in our understanding of the mechanistic basis of ASD because of GATA6 mutations.

Homozygous GATA6 null mice die before cardiac development, whereas heterozygous GATA6 null mice show no obvious cardiac phenotypes. ${ }^{29,30}$ Meanwhile, we identified the same heterozygous, non-synonymous mutation in three patients with differing clinical manifestations. One important reason for this difference between the two species is likely different gene dosage sensitivities in human and mice. ${ }^{1}$ Humans are more vulnerable to subtle genetic defects but are able to survive with various types of cardiac abnormalities, unlike the embryonic lethality commonly seen in mice with the same cardiac defects. ${ }^{31}$ The underlying mechanism of the different phenotypes of the identified GATA6 mutation are unclear, but environmental factors, epigenetic factors and other mediators may be responsible.

In conclusion, we identified a novel GATA6 mutation in patients with TOF and ASDs. The identified GATA6 mutation causes impaired biological function of the protein, suggesting that it is likely to have a role in the pathogenesis of CHD. Studies on the impact of this GATA6 mutation on specific cardiac defects will be necessary to further elucidate its impact on cardiovascular development.

\section{ACKNOWLEDGEMENTS}

This work was supported by the National Science Fund of China (30425016, 30330290 and 30470961), the '973' Program Fund of China (2007CB512100), the ' 863 ' Program Fund of China (2007AA02Z438), the Program Fund for Outstanding Medical Academic Leaders of Shanghai, the Program Fund for Shanghai Subject Chief Scientists, the Yangtze Scholars Program Fund by the Ministry of Education of China, the Program Fund for Innovative Research Teams by the Ministry of Education of China (Y-HC).

1 Bruneau, B. G. The developmental genetics of congenital heart disease. Nature 451, 943-948 (2008)

2 Pierpont, M. E., Basson, C. T., Benson, D. W. Jr., Gelb, B. D., Giglia, T. M., Goldmuntz, E. et al. Genetic basis for congenital heart defects: current knowledge: a scientific statement from the American Heart Association Congenital Cardiac Defects Committee, Council on Cardiovascular Disease in the Young: endorsed by the American Academy of Pediatrics. Circulation 115, 3015-3038 (2007).

3 Basson, C. T., Bachinsky, D. R., Lin, R. C., Levi, T., Elkins, J. A., Soults, J. et al. Mutations in human TBX5 [corrected] cause limb and cardiac malformation in HoltOram syndrome. Nat. Genet. 15, 30-35 (1997).

4 Garg, V., Kathiriya, I. S., Barnes, R., Schluterman, M. K., King, I. N., Butler, C. A. et al. GATA4 mutations cause human congenital heart defects and reveal an interaction with TBX5. Nature 424, 443-447 (2003).

5 Li, Q. Y., Newbury-Ecob, R. A., Terrett, J. A., Wilson, D. I., Curtis, A. R., Yi, C. H. et al. Holt-Oram syndrome is caused by mutations in TBX5, a member of the Brachyury (T) gene family. Nat. Genet. 15, 21-29 (1997).

6 Schott, J. J., Benson, D. W., Basson, C. T., Pease, W., Silberbach, G. M., Moak, J. P. et al. Congenital heart disease caused by mutations in the transcription factor NKX2-5. Science (New York, N.Y.) 281, 108-111 (1998).

7 Molkentin, J. D. The zinc finger-containing transcription factors GATA-4, -5 , and -6 . Ubiquitously expressed regulators of tissue-specific gene expression. J. Biol. Chem. 275, 38949-38952 (2000).

8 Patient, R. K. \& McGhee, J. D. The GATA family (vertebrates and invertebrates). Curr. Opin. Genet. Dev. 12, 416-422 (2002).

9 Peterkin, T., Gibson, A., Loose, M. \& Patient, R. The roles of GATA-4, -5 and -6 in vertebrate heart development. Semin. Cell. Dev. Biol. 16, 83-94 (2005).

10 Hirayama-Yamada, K., Kamisago, M., Akimoto, K., Aotsuka, H., Nakamura, Y., Tomita, H. et al. Phenotypes with GATA4 or NKX2.5 mutations in familial atrial septal defect. Am. J. Med. Genet. A 135, 47-52 (2005).

11 Nemer, G., Fadlalah, F., Usta, J., Nemer, M., Dbaibo, G., Obeid, M. et al. A novel mutation in the GATA4 gene in patients with Tetralogy of Fallot. Hum. Mutat. 27, 293-294 (2006).

12 Okubo, A., Miyoshi, O., Baba, K., Takagi, M., Tsukamoto, K., Kinoshita, A. et al. A novel GATA4 mutation completely segregated with atrial septal defect in a large Japanese family. J. Med. Genet. 41, e97 (2004). 
13 Posch, M. G., Perrot, A., Schmitt, K., Mittelhaus, S., Esenwein, E. M., Stiller, B. et al. Mutations in GATA4, NKX2.5, CRELD1, and BMP4 are infrequently found in patients with congenital cardiac septal defects. Am. J. Med. Genet. A 146A, 251-253 (2008).

14 Sarkozy, A., Conti, E., Neri, C., D’Agostino, R., Digilio, M. C., Esposito, G. et al. Spectrum of atrial septal defects associated with mutations of NKX2.5 and GATA4 transcription factors. J. Med. Genet. 42, e16 (2005).

15 Schluterman, M. K., Krysiak, A. E., Kathiriya, I. S., Abate, N., Chandalia, M., Srivastava, D. et al. Screening and biochemical analysis of GATA4 sequence variations identified in patients with congenital heart disease. Am. J. Med. Genet. A 143A, 817823 (2007)

16 Tomita-Mitchell, A., Maslen, C. L., Morris, C. D., Garg, V. \& Goldmuntz, E. GATA4 sequence variants in patients with congenital heart disease. J. Med. Genet. 44, 779 783 (2007)

17 Zhang, W., Li, X., Shen, A., Jiao, W., Guan, X. \& Li, Z. GATA4 mutations in 486 Chinese patients with congenital heart disease. Eur. J. Med. Genet. 51, 527-535 (2008).

18 Lepore, J. J., Mericko, P. A., Cheng, L., Lu, M. M., Morrisey, E. E. \& Parmacek, M. S. GATA- 6 regulates semaphorin $3 C$ and is required in cardiac neural crest for cardiovascular morphogenesis. J. Clin. Invest. 116, 929-939 (2006).

19 Maitra, M., Schluterman, M. K., Nichols, H. A., Richardson, J. A., Lo, C. W., Srivastava, D. et al. Interaction of Gata4 and Gata6 with Tbx5 is critical for normal cardiac development. Dev. Biol. 326, 368-377 (2009).

20 Xin, M., Davis, C. A., Molkentin, J. D., Lien, C. L., Duncan, S. A., Richardson, J. A. et al. A threshold of GATA4 and GATA6 expression is required for cardiovascular development. Proc. Natl Acad. Sci. USA 103, 11189-11194 (2006).

21 Zhao, R., Watt, A. J., Battle, M. A., Li, J., Bondow, B. J. \& Duncan, S. A. Loss of both GATA4 and GATA6 blocks cardiac myocyte differentiation and results in acardia in mice. Dev. Biol. 317, 614-619 (2008).
22 Kodo, K., Nishizawa, T., Furutani, M., Arai, S., Yamamura, E., Joo, K. et al. GATA6 mutations cause human cardiac outflow tract defects by disrupting semaphorin-plexin signaling. Proc. Natl Acad. Sci. USA 106, 13933-13938 (2009).

23 Li, H. H., Kedar, V., Zhang, C., McDonough, H., Arya, R., Wang, D. Z. et al. Atrogin-1/ muscle atrophy F-box inhibits calcineurin-dependent cardiac hypertrophy by participating in an SCF ubiquitin ligase complex. J. Clin. Invest. 114, 1058-1071 (2004).

24 Fedak, P. W., Verma, S., David, T. E., Leask, R. L., Weisel, R. D. \& Butany, J. Clinical and pathophysiological implications of a bicuspid aortic valve. Circulation 106, 900904 (2002).

25 Nakamura, T., Colbert, M. C. \& Robbins, J. Neural crest cells retain multipotential characteristics in the developing valves and label the cardiac conduction system. Circ. Res. 98, 1547-1554 (2006).

26 Martin, L. J., Ramachandran, V., Cripe, L. H., Hinton, R. B., Andelfinger, G., Tabangin, M. et al. Evidence in favor of linkage to human chromosomal regions $18 q, 5 q$ and $13 q$ for bicuspid aortic valve and associated cardiovascular malformations. Hum. Genet. 121, 275-284 (2007).

27 Morrisey, E. E., Ip, H. S., Tang, Z. \& Parmacek, M. S. GATA-4 activates transcription via two novel domains that are conserved within the GATA-4/5/6 subfamily. J. Biol. Chem. 272, 8515-8524 (1997).

28 Charron, F., Paradis, P., Bronchain, O., Nemer, G. \& Nemer, M. Cooperative interaction between GATA-4 and GATA-6 regulates myocardial gene expression. Mol. Cell. Biol. 19, 4355-4365 (1999).

29 Koutsourakis, M., Langeveld, A., Patient, R., Beddington, R. \& Grosveld, F. The transcription factor GATA6 is essential for early extraembryonic development. Development (Cambridge, England) 126, 723-732 (1999).

30 Morrisey, E. E., Tang, Z., Sigrist, K., Lu, M. M., Jiang, F., Ip, H. S. et al. GATA6 regulates HNF4 and is required for differentiation of visceral endoderm in the mouse embryo. Genes. Dev. 12, 3579-3590 (1998).

31 Srivastava, D. \& Olson, E. N. A genetic blueprint for cardiac development. Nature 407, 221-226 (2000). 